\title{
Fluid flow and conjugated heat transfer in arbitrarily shaped channels via single domain formulation and integral transforms
}

\author{
Diego C. Knupp ${ }^{\mathrm{a}}$, Renato M. Cotta ${ }^{\mathrm{b}, \mathrm{,},}$, Carolina P. Naveira-Cotta ${ }^{\mathrm{b}}$ \\ a Instituto Politécnico, Universidade do Estado do Rio de Janeiro, IPRJ/UERJ, Patrícia Oliva Soares Laboratory of Experimentation and Numerical Simulation in Heat and Mass \\ Transfer, LEMA, Dept. of Mechanical Engineering and Energy, Nova Friburgo, RJ CEP 28625-570, Brazil \\ ${ }^{\mathrm{b}}$ Laboratory of Nano- and Microfluidics and Microsystems, LabMEMS, Mechanical Engineering Dept. (PEM) E Nanoengineering Dept. (PENT) - POLIECOPPE, UFRJ, \\ Universidade Federal do Rio de Janeiro, Cidade Universitária, Cx. Postal 68503, Rio de Janeiro, RJ CEP 21945-970, Brazil

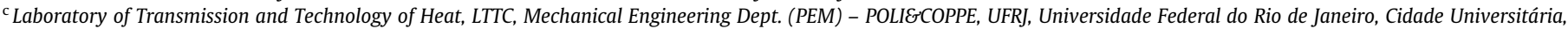 \\ Cx. Postal 68503, Rio de Janeiro, RJ CEP 21945-970, Brazil
}

\section{A R T I C L E I N F O}

\section{Article history:}

Received 19 August 2014

Accepted 3 November 2014

Available online 26 November 2014

\section{Keywords:}

Internal flow

Single domain formulation

Hybrid methods

Integral transforms

Conjugated heat transfer

Microchannels

Micro-heat exchangers

\begin{abstract}
A B S T R A C T
The present work advances a recently introduced approach based on combining the Generalized Integral Transform Technique (GITT) and a single domain reformulation strategy, aimed at providing hybrid numerical-analytical solutions to convection-diffusion problems in complex physical configurations and irregular geometries. The methodology has been previously considered in the analysis of conjugated conduction-convection heat transfer problems, simultaneously modeling the heat transfer phenomena at both the fluid streams and the channels walls, by making use of coefficients represented as space variable functions with abrupt transitions occurring at the fluid-wall interfaces. The present work is aimed at extending this methodology to deal with both fluid flow and conjugated heat transfer within arbitrarily shaped channels and complex multichannel configurations, so that the solution of a cumbersome system of coupled partial differential equations defined for each individual sub-domain of the problem is avoided, with the proposition of the single-domain formulation. The reformulated problem is integral transformed through the adoption of eigenvalue problems containing the space variable coefficients, which provide the basis of the eigenfunction expansions and are responsible for recovering the transitional behavior among the different regions in the original formulation. For demonstration purposes, an application is first considered consisting of a microchannel with an irregular cross-section shape, representing a typical channel micro-fabricated through laser ablation, in which heat and fluid flow are investigated, taking into account the conjugation with the polymeric substrate. Then, a complex configuration consisting of multiple irregularly shaped channels is more closely analyzed, in order to illustrate the flexibility and robustness of the advanced hybrid approach. In both cases, the convergence behavior of the proposed expansions is presented and critical comparisons against purely numerical approaches are provided.
\end{abstract}

() 2014 Elsevier Ltd. All rights reserved.

\section{Introduction}

Along the last few decades, the research efforts towards the development of numerical solutions of conjugated heat and fluid flow formulations [1-3] has been quite remarkable. The level of scientific maturity in this area is quite evident in the quite general state-of-the-art computer codes, flexible enough to handle most engineering applications, such as [4,5]. Nevertheless, the solution

\footnotetext{
* Corresponding author at: Laboratory of Transmission and Technology of Heat, LTTC, Mechanical Engineering Dept. (PEM) - POLI\&COPPE, UFRJ, Universidade Federal do Rio de Janeiro, Cidade Universitária, Cx. Postal 68503, Rio de Janeiro, RJ CEP 21945-970, Brazil.
}

of the related set of mass, momentum and energy balance equations remains an important and still growing research area due to its relevant technological implications. While the main research front has always been driven by developments on discrete computational approaches, a few research groups devoted their attention to the parallel development of hybrid numerical-analytical techniques, aimed at reducing overall computational costs and achieving more robust accuracy control schemes, which to some extent resulted in a revival of some classical analytical methods. The Generalized Integral Transform Technique (GITT) [6-9] is a typical example of such hybrid methods, which employs eigenfunction expansions to analytically express the sought potential in all but one independent variable, and numerically (or eventually 


\section{Nomenclature}

$d \quad$ linear dissipation operator

$K \quad$ diffusion operator coefficient

$k \quad$ dimensionless thermal conductivity in problem (11)

$L_{x}, L_{y}, L_{z}$ domain length in $x, y$, and $z$ directions, respectively

$M \quad$ truncation order of the eigenvalue problem solution

$N \quad$ truncation order of the velocity field expansions

$p \quad$ pressure field

$P \quad$ source term

$r \quad$ radial coordinate in the exact analytical solution of a circular tube

$R \quad$ radius of the circular channel

$T$ dimensionless potential

$t \quad$ dimensionless time variable

$u \quad$ velocity field component in the longitudinal direction

$w \quad$ transient operator coefficient

$x, y \quad$ transversal coordinates

$z \quad$ longitudinal coordinate

\section{Greek letters}

$\Delta p \quad$ pressure difference between outlet and inlet

$\alpha, \beta \quad$ boundary conditions coefficients

$\phi \quad$ source term in boundary conditions

$\mu, \eta \quad$ eigenvalues corresponding to eigenfunctions $\psi$ and $\chi$, respectively

$v \quad$ kinematic viscosity

$\rho \quad$ density

$\psi, \chi \quad$ eigenfunctions

Subscripts and superscripts

- $\quad$ integral transformation

$\sim \quad$ normalized eigenfunction

$f \quad$ quantity corresponding to the fluid regions

$i, j \quad$ order of eigenquantities

$s \quad$ quantity corresponding to the solid regions analytically) solve the transformed ordinary (or partial) differential system in the remaining independent variable (or variables).

The application of the integral transform approach to either conjugated heat transfer problems with wall lumped models [10-13] or internal forced convection problems in regularly shaped channels [14-17] is well documented in a number of contributions along the last two decades, mostly addressed to benchmarking and verification in the realm of classical test problems, profiting of the method's intrinsic characteristic of finding solutions with automatic global error control. In addition, the GITT approach has been advanced, since the early phases of its development, in handling diffusion problems within irregular domains, including fully developed and developing flow within irregularly shaped channels [1824], by considering integral transformations with space dependent eigenvalues.

Convective heat transfer within irregularly shaped ducts is found in several practical applications, for instance, in the flow of liquids in chemical processing plants, air flow in cooling, heating and ventilation units, and cooling of electronic equipment. In the context of microfluidics and microsystems, besides the cooling of electronic components, one may also recall the crucial importance of convection within microchannels in various applications that require micro-heat exchangers, micro-heat spreaders, and microreactors $[25,26]$. In some of these situations the flow in irregular geometries must be tackled, either because of the complex geometries involved, or as a result of micro-fabrication irregularities. In fact, for the conception and design of thermal microsystems, several works have been devoted to building and revisiting reliable models and solution methodologies capable of describing the physical phenomena that take place in such microscale heat and fluid flow problems $[27,28]$.

Motivated by the theoretical discussion in [29], the experimental and theoretical results in [13] have shown the importance of considering heat conduction along microchannels walls in certain situations, leading to a conjugated conduction-internal convection heat transfer problem, in this case handled through integral transformation with an improved wall lumping procedure, which yielded results in better agreement with the reported experimental data. More recently, the reformulation of conjugated conductionconvection problems as a single region model has been proposed [30], accounting for the local heat transfer at both the fluid flow and the channel wall regions, by making use of coefficients represented as space-variable functions, with abrupt transitions occur- ring at the fluid-solid interfaces. In this single region formulation, the mathematical model incorporates the information concerning the multiple original domains of the problem. The excellent agreement between the proposed methodology and the exact solution for a fairly simple test case presented in [30], motivated the extension of the approach, which has been demonstrated to be extendable to the solution of more involved problems, for example when dealing with axial conduction both at the channel walls and in the fluid stream [31], when taking into account the heat conduction to the upstream region of the heat exchange section [32], which may be important in problems with low Péclet numbers, in fully three dimensional conjugated heat transfer problems [33], and in a real application of multiple circular microchannels etched onto a nanocomposite substrate [34].

In these recent contributions [30-34] on conjugated heat transfer analysis, it is always considered that the duct has a regular shape such as parallel plates, circular tubes or rectangular channels, and that the fully developed velocity field is known from available analytical expressions. However, this novel approach is flexible enough to deal with arbitrarily shaped channels, even in a more direct way than previously proposed in the solution of diffusion problems through integral transforms [18,21]. Thus, in the present work we advance the combined integral transforms and single domain formulation approach to solve the flow and energy equations for conjugated heat transfer within a channel with arbitrarily shaped cross-section and its associated solid substrate. Hence, both the fully developed velocity field and temperature distributions in the fluid and the solid, are represented as eigenfunction expansions. The idea is to represent the complex domain as a simpler single domain problem with spatially variable coefficients, which account for the transitions between the original problem regions. Following the solution methodology developed in previous works [30-34], the GITT is then employed in the direct integral transformation of the problem with space-variable coefficients, as well as on the solution of the associated eigenvalue problem $[35,36]$, in order to transform the original differential eigenvalue problems into algebraic eigensystems, to be readily solved through available built-in routines, such as in the Mathematica system [37].

In order to verify the adequacy of the proposed approach, first a test case is investigated, which consists of the flow inside a channel with an arbitrary cross-section shape, inspired by an actual situation of micro-fabrication through laser ablation. In addition, a more complex multichannel configuration is considered more closely, 
allowing for an illustration of the flexibility and capability of the proposed hybrid methodology. The derived fully developed velocity fields and the temperature distributions, from both conjugated heat transfer formulations, are critically compared against purely numerical solutions obtained through the finite element method, employing the commercial CFD solver COMSOL Multiphysics.

\section{Single domain formulation and integral transforms solution}

A fairly general problem formulation is now analyzed and solved, which may be applied to different physical situations, not only to the specific convection-conduction conjugated problems here of major interest. Thus, consider a general transient diffusion or convection-diffusion problem defined in a complex multidimensional configuration that is represented by $n_{V}$ different sub-regions with volumes $V_{l}, l=1,2, \ldots, n_{V}$, with potential and flux continuity at the interfaces among themselves, as illustrated in Fig. 1(a). We consider that a certain number of potentials are to be calculated in each sub-region, $T_{k, l}(\mathbf{x}, t), k=1,2, \ldots, n_{T}$, for instance temperature, concentrations, velocity components, and pressure, governed in the corresponding sub-region through a fairly general formulation including general equation and boundary source terms, respectively, $P_{k, l}(\mathbf{x}, t, \mathbf{T})$ and $\phi_{k, l}(x, t, \mathbf{T})$ :

$$
\begin{aligned}
w_{k, l}(\mathbf{x}) \frac{\partial T_{k, l}(\mathbf{x}, t)}{\partial t}= & \nabla \cdot\left(K_{k, l}(\mathbf{x}) \nabla T_{k, l}(\mathbf{x}, t)\right)-d_{k, l}(\mathbf{x}) T_{k, l}(\mathbf{x}, t) \\
& +P_{k, l}(\mathbf{x}, t, \mathbf{T}), \quad \mathbf{x} \in V_{l}, t>0, k=1,2, \ldots, n_{T}, \\
l= & 1,2, \ldots, n_{V}
\end{aligned}
$$

with initial, interface and boundary conditions given, respectively, by

$$
\begin{aligned}
& T_{k, l}(\mathbf{x}, 0)=f_{k, l}(\mathbf{x}), \quad \mathbf{x} \in V_{l} \\
& T_{k, l}(\mathbf{x}, t)=T_{k, m}(\mathbf{x}, t), \quad \mathbf{x} \in S_{l, m}, t>0 \\
& K_{k, l}(\mathbf{x}) \frac{\partial T_{k, l}(\mathbf{x}, t)}{\partial \mathbf{n}}=K_{k, m}(\mathbf{x}) \frac{\partial T_{k, m}(\mathbf{x}, t)}{\partial \mathbf{n}}, \quad \mathbf{x} \in S_{l, m}, t>0 \\
& {\left[\alpha_{k, l}(\mathbf{x})+\beta_{k, l}(\mathbf{x}) K_{k, l}(\mathbf{x}) \frac{\partial}{\partial \mathbf{n}}\right] T_{k, l}(\mathbf{x}, t)=\phi_{k, l}(\mathbf{x}, t, \mathbf{T}), \quad \mathbf{x} \in S_{l}, t>0}
\end{aligned}
$$

where $\mathbf{n}$ denotes the outward-drawn normal to the interfaces, $S_{l, m}$, and external surfaces, $S_{l}$. The source terms may incorporate non-linear coefficients, convective terms, or even partial derivatives with respect to a certain spatial coordinate, chosen not to be integral

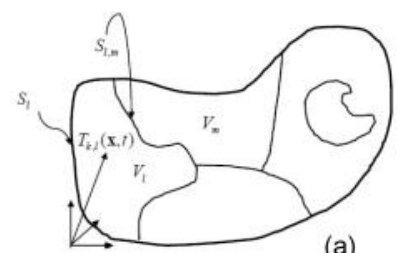

(a)

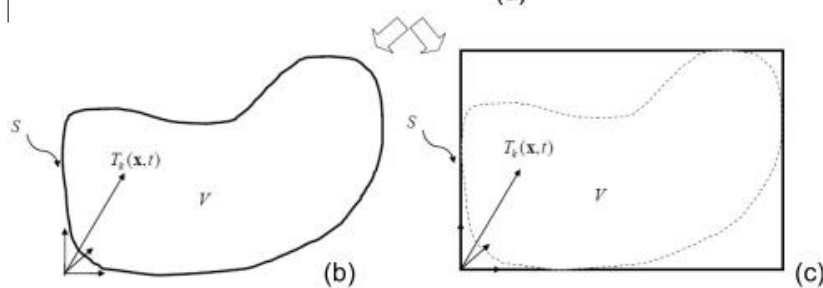

(c)

Fig. 1. (a) Diffusion or convection-diffusion in a complex multidimensional configuration with $n_{V}$ sub-regions; (b) Single domain representation keeping the original overall domain; (c) Single domain representation considering a regular overall domain that envelopes the original one. transformed, such as when a partial transformation scheme is adopted in the GITT solution [38]. In the case of partial transformation, the position vector $\mathbf{x}$ and corresponding regions $V_{l}$ are defined only in terms of the spatial coordinates to be eliminated by integral transformation. Also, the appropriate boundary conditions on this specific remaining coordinate have to be provided, apart from those specified for the coordinates that compose vector $\mathbf{x}$ [38].

The Generalized Integral Transform Technique (GITT) [6-9] can be directly applied to solve system (1) above, either by constructing an individual eigenfunction expansion basis for each potential, with adequate implicit filtering, and then coupling all the transformed systems and potentials for each sub-region, or by constructing a multiregion eigenvalue problem that couples all of the sub-regions into a single set of eigenvalues, which in general involves cumbersome computations in multidimensional applications. However, in this case, one single transformed system and one single set of transformed potentials is obtained by employing the appropriate orthogonality property.

Fig. 1 provides two possibilities for representation of the single domain, either by keeping the original overall domain after definition of the space variable coefficients, as shown in Fig. 1(b), or, if desired, by considering a regular overall domain that envelopes the original one, as shown in Fig. 1(c). Irregular domains can be directly integral transformed, as demonstrated in several previous works, for example in refs. [18-24] and, in principle, there is no need to consider the second representation possibility pointed out above. However, some computational advantages may be achieved by enveloping the original irregular domain by a simple regular region, especially in the context of automatic solution procedures, such as when using the UNIT (UNified Integral Transforms) algorithm and open source code [38,39].

Therefore, as already demonstrated in the analysis of a few specific conjugated problems [30-34], it is possible to rewrite problem (1) as a single domain formulation with space variable coefficients and source terms, given by:

$$
\begin{aligned}
w_{k}(\mathbf{x}) \frac{\partial T_{k}(\mathbf{x}, t)}{\partial t}= & \nabla \cdot\left(K_{k}(\mathbf{x}) \nabla T_{k}(\mathbf{x}, t)\right)-d_{k}(\mathbf{x}) T_{k}(\mathbf{x}, t)+P_{k}(\mathbf{x}, t, \mathbf{T}), \\
& \mathbf{x} \in V, t>0
\end{aligned}
$$

with initial and boundary conditions given, respectively, by

$T_{k}(\mathbf{x}, 0)=f_{k}(\mathbf{x}), \quad \mathbf{x} \in V$

$\left[\alpha_{k}(\mathbf{x})+\beta_{k}(\mathbf{x}) K_{k}(\mathbf{x}) \frac{\partial}{\partial \mathbf{n}}\right] T_{k}(\mathbf{x}, t)=\phi_{k}(\mathbf{x}, t, \mathbf{T}), \quad \mathbf{x} \in S, t>0$

where

$V=\sum_{l=1}^{n_{V}} V_{l}, \quad S=\sum_{l=1}^{n_{V}} S_{l}$

and where the potentials vector is given by

$\mathbf{T}=\left\{T_{1}, T_{2}, \ldots, T_{k}, \ldots, T_{n_{T}}\right\}$

The space variable coefficients in Eq. (2), besides the new equation and boundary source terms and initial conditions, now without the subscript $l$ for the sub-regions $V_{l}$, incorporate the abrupt transitions among the different sub-regions and permit the representation of system (1) as a single domain formulation, to be directly handled by integral transforms.

The formal integral transform solution of problem (2) requires the proposition of eigenfunction expansions for the associated potentials. The linear situation, when the equation and boundary source terms in Eq. (2) do not depend on T, i.e. with $P_{k}(\mathbf{x}, t, \mathbf{T}) \equiv$ $P_{k}(\mathbf{x}, t)$ and $\phi_{k}(x, t, \mathbf{T}) \equiv \phi_{k}(\mathbf{x}, t)$, allows for an exact solution via the 
classical integral transform approach [6], and naturally leads to the eigenvalue problems to be preferred in the analysis of the nonlinear situation. They appear in the direct application of the separation of variables methodology to the linear homogeneous purely diffusive version of the proposed problem. Thus, the suggested set of uncoupled auxiliary problems is given by:

$$
\begin{aligned}
& \nabla \cdot\left(K_{k}(\mathbf{x}) \nabla \psi_{k i}(\mathbf{x})\right)+\left[\mu_{k i}^{2} w_{k}(\mathbf{x})-d_{k}(\mathbf{x})\right] \psi_{k i}(\mathbf{x})=0, \quad \mathbf{x} \in V \\
& {\left[\alpha_{k}(\mathbf{x})+\beta_{k}(\mathbf{x}) K_{k}(\mathbf{x}) \frac{\partial}{\partial \mathbf{n}}\right] \psi_{k i}(\mathbf{x})=0, \quad \mathbf{x} \in S}
\end{aligned}
$$

where the eigenvalues, $\mu_{k i}$, and corresponding eigenfunctions, $\psi_{k i}(\mathbf{x})$, are assumed to be known from application of the GITT itself in the solution of Sturm-Liouville type problems, by reducing Eqs. (3a) and (3b) to standard algebraic eigenvalue problems (Appendix A). In fact, the written form of Eq. (2) already reflects the choice of eigenvalue problems given by Eq. (3), via prescription of the linear coefficients in both the equations and boundary conditions, since any remaining term in the original formulation is directly incorporated into the general nonlinear source terms, without loss of generality. Problem (3) allows, through the associated orthogonality properties of the eigenfunctions, the definition of the following integral transform pairs:

$\bar{T}_{k i}(t)=\int_{V} w_{k}(\mathbf{x}) \tilde{\psi}_{k i}(\mathbf{x}) T_{k}(\mathbf{x}, t) \mathrm{d} V, \quad$ transforms

$T_{k}(\mathbf{x}, t)=\sum_{i=1}^{\infty} \tilde{\psi}_{k i}(\mathbf{x}) \bar{T}_{k, i}, \quad$ inverses

where the symmetric kernels $\tilde{\psi}_{k i}(x)$ are given by

$\tilde{\psi}_{k i}(\mathbf{x})=\frac{\psi_{k i}(\mathbf{x})}{\sqrt{N_{k i}}} ; \quad N_{k i}=\int_{V} w_{k}(\mathbf{x}) \psi_{k i}^{2}(\mathbf{x}) \mathrm{d} V$

with $N_{k i}$ being the normalization integrals.

The integral transformation of Eq. (2a) is accomplished by applying the operator $\int_{V} \tilde{\psi}_{k i}(\mathbf{x})(\cdot) \mathrm{d} V$ and making use of the boundary conditions given by Eqs. (2c) and (3b), yielding:

$\frac{\partial \bar{T}_{k i}(t)}{\partial t}+\mu_{k i}^{2} \bar{T}_{k i}(t)=\bar{g}_{k i}(t, \overline{\mathbf{T}})+\bar{b}_{k i}(t, \overline{\mathbf{T}}), \quad i=1,2, \ldots, \quad t>0$,
$k=1,2, \ldots, n_{T}$

where the first transformed source term $\bar{g}_{k i}(t, \overline{\mathbf{T}})$ is due to the integral transformation of the equation source term, and the second, $\bar{b}_{k i}(t, \overline{\mathbf{T}})$, is due to the contribution of the boundary source term:

$\bar{g}_{k i}(t, \overline{\mathbf{T}})=\int_{V} \tilde{\psi}_{k i}(\mathbf{x}) P_{k}(\mathbf{x}, t, \mathbf{T}) d V$

$\bar{b}_{k i}(t, \overline{\mathbf{T}})=\int_{S} K_{k}(\mathbf{x})\left[\tilde{\psi}_{k i}(\mathbf{x}) \frac{\partial T_{k}(\mathbf{x}, t)}{\partial \mathbf{n}}-T_{k}(\mathbf{x}, t) \frac{\partial \tilde{\psi}_{k i}(\mathbf{x})}{\partial \mathbf{n}}\right] d s$

The boundary conditions contribution may also be expressed in terms of the boundary source terms, after manipulating Eqs. (2c) and (3b), to yield:

$\bar{b}_{k i}(t, \overline{\mathbf{T}})=\int_{S} \phi_{k}(\mathbf{x}, t, \mathbf{T})\left[\frac{\tilde{\psi}_{k i}(\mathbf{x})-K_{k}(\mathbf{x}) \frac{\partial \tilde{\psi}_{k i}(\mathbf{x})}{\partial \mathbf{n}}}{\alpha_{k}(\mathbf{x})+\beta_{k}(\mathbf{x})}\right] d s$

The initial conditions given by Eq. (2b) are transformed through the operator $\int_{V} w_{k}(\mathbf{x}) \tilde{\psi}_{k i}(\mathbf{x})(\cdot) d V$, to provide:

$\bar{T}_{k i}(0)=\bar{f}_{k i} \equiv \int_{V} w_{k}(\mathbf{x}) \tilde{\psi}_{k i}(\mathbf{x}) f_{k}(\mathbf{x}) d V$

Under the total transformation scheme, more frequently employed in the GITT literature [38,39], Eq. (5) form an infinite coupled system of nonlinear ordinary differential equations (ODE's) for the transformed potentials, $\bar{T}_{k i}(t)$. If one chooses to adopt the partial transformation scheme [38], the transformed source term is a function also of the coordinate chosen not to be integral transformed and its corresponding differential operators grouped into the source term. In this case, the resulting transformed problem (5) is a system of partial differential equations (PDE's), and the corresponding transformed boundary conditions in this untransformed spatial coordinate have to be obtained through the same integral transformation operator employed for the initial condition (5e). The resulting infinite coupled nonlinear system of differential equations, either ordinary or partial, is unlikely to be analytically solvable. Nevertheless, reliable algorithms are readily available to numerically handle this system, after truncation of the infinite coupled system to a sufficiently large finite order. For instance, the Mathematica system [38,39] provides the built-in routine NDSolve for solving differential systems such as the ones here obtained, under automatic absolute and relative error control. It must also be recalled that for a $t$-independent formulation, such as for a steady state convection-diffusion problem, the corresponding transformed system (5a) for the total transformation, results in a linear or nonlinear algebraic problem to be numerically solved for the transformed potentials.

Once the transformed potentials have been numerically computed, the inversion formula, Eq. (4b), can be recalled to yield the potential field representation at any desired position $\mathbf{x}$ and time $t$, and again, for a partial transformation scheme, also as a function of the untransformed spatial coordinate. The formal solutions above derived provide the basic working expressions for the integral transform method. However, it should be highlighted that for an improved computational performance, it is always recommended to reduce the importance of the equation and boundary source terms, for instance through analytical filtering, in order to enhance the eigenfunction expansions convergence behavior [6-9].

\section{Application to fluid flow and conjugated heat transfer in irregularly shaped ducts}

\subsection{Flow problem}

Consider steady-state laminar incompressible fully developed flow of a Newtonian fluid in a channel with arbitrarily-shaped, but longitudinally constant, cross-section, as schematically shown in Fig. 2. The momentum equation in the longitudinal direction $(z)$, formulated as a single region model, is given by:

$\frac{\partial}{\partial x}\left(v(x, y) \frac{\partial u(x, y)}{\partial x}\right)+\frac{\partial}{\partial y}\left(v(x, y) \frac{\partial u(x, y)}{\partial y}\right)-\frac{C(x, y)}{\rho(x, y)}=0$

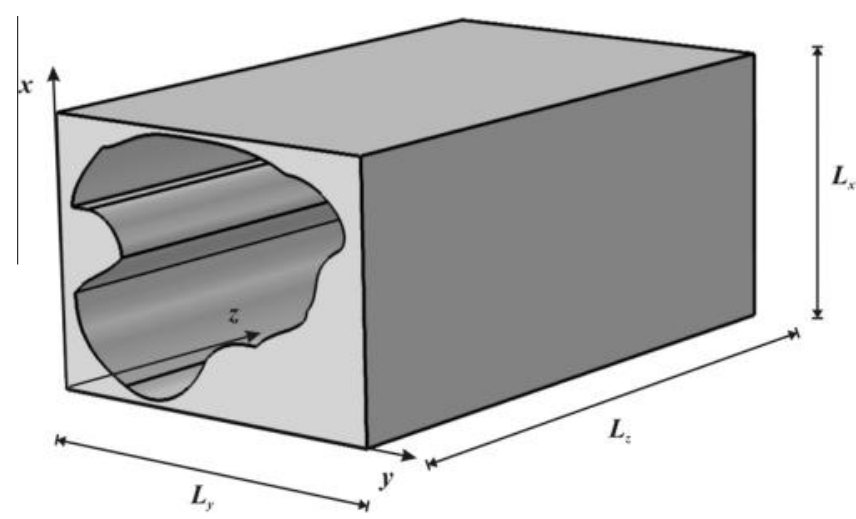

Fig. 2. Schematic representation of a channel with arbitrarily shaped cross section in a rectangular domain. 
$u(0, y)=u\left(L_{x}, y\right)=u(x, 0)=u\left(x, L_{y}\right)=0$

with:

$C(x, y)= \begin{cases}\frac{\partial p}{\partial z}=\Delta p / L_{z}, & \text { in the fluid region } \\ 0, & \text { in the solid wall region }\end{cases}$

where the kinematic viscosity and density, $v(x, y)$ and $\rho(x, y)$, are given, respectively, by:

$v(x, y)= \begin{cases}v_{f}, & \text { in the fluid region } \\ v_{s} \rightarrow \infty, & \text { in the solid wall region }\end{cases}$

$\rho(x, y)= \begin{cases}\rho_{f}, & \text { in the fluid region } \\ \rho_{s}, & \text { in the solid wall region }\end{cases}$

where $v_{f}$ and $\rho_{f}$ stand for the kinematic viscosity and density of the fluid, and $v_{s}$ and $\rho_{s}$ for the solid. For $v_{s}$ it suffices to choose a sufficiently large value, when the value for $\rho_{s}$ will no longer affect the final result and the calculated velocities in the solid region will result to be zero, as physically expected.

Following the Generalized Integral Transform Technique formalism, the transform/inverse pair for solving problem (6) is defined as follows:

transform : $\quad \bar{u}_{i}=\int_{0}^{L_{y}} \int_{0}^{L_{x}} \tilde{\chi}_{i}(x, y) u(x, y) d x d y$

inverse : $\quad u(x, y)=\sum_{i=1}^{\infty} \tilde{\chi}_{i}(x, y) \bar{u}_{i}$

with:

$\tilde{\chi}_{i}(x, y)=\frac{\chi_{i}(x, y)}{\sqrt{N_{i}}}$

$N_{i}=\int_{0}^{L_{x}} \int_{0}^{L_{y}} \chi_{i}^{2}(x, y) d x d y$

where the eigenfunctions $\tilde{\chi}_{i}(x, y)$ and corresponding eigenvalues $\eta_{i}$ are calculated from the solution of a properly chosen eigenvalue problem. As discussed in Section 2, adopting the eigenvalue problem obtained by directly applying separation of variables to problem (6) is desirable, so that all the information concerning the transition of the two original domains may be represented within the eigenfunctions. Therefore, we consider the following eigenvalue problem:

$\frac{\partial}{\partial x}\left(v(x, y) \frac{\partial \chi_{i}(x, y)}{\partial x}\right)+\frac{\partial}{\partial y}\left(v(x, y) \frac{\partial \chi_{i}(x, y)}{\partial y}\right)+\eta_{i}^{2} \chi_{i}=0$

$\chi(0, y)=\chi\left(L_{x}, y\right)=\chi(x, 0)=\chi\left(x, L_{y}\right)=0$

Problem (9) does not allow for an explicit analytic solution, but the Generalized Integral Transform Technique itself can be employed in order to provide a hybrid numerical-analytical solution, by reducing Eqs. (9a) and (9b) to a standard algebraic eigenvalue problem (see Appendix A). Once this solution is made available, problem (6) becomes fully transformable when operated with $\int_{0}^{L_{y}} \int_{0}^{L_{x}} \tilde{\chi}_{i}(x, y)(\cdot) d x d y$, yielding a linear decoupled algebraic problem for the transformed velocities, with the analytical solution:

$\bar{u}_{i}=\frac{1}{\eta_{i}^{2}} \int_{0}^{L_{y}} \int_{0}^{L_{x}} \tilde{\chi}_{i}(x, y) \frac{C(x, y)}{\rho(x, y)} d x d y, \quad i=1,2, \ldots$

The transformed potentials $\bar{u}_{i}$ given by Eq. (10) above can be readily substituted into the inverse formula given by Eq. (8b), yielding an analytic representation for the velocity field $u(x, y)$.

\subsection{Conjugated heat transfer problem}

Considering again Fig. 2 as the single domain representation, we now deal with the conjugated heat transfer problem involving thermally developing fluid flow within the irregular channel and the surrounding solid walls. It should be clarified that the bounding domain for the analysis of the flow problem is not required to be the same as the actual physical domain in the conjugated heat transfer analysis, including the whole solid region, since the objective of proposing a bounding domain for the flow problem is merely to simplify the solution of the original problem, by performing the integral transformation over a regular domain. The transient single domain formulation for the energy balance is then written as:

$$
\begin{aligned}
w(x, y) \frac{\partial T(x, y, z, t)}{\partial t}= & \frac{\partial}{\partial x}\left(k(x, y) \frac{\partial T}{\partial x}\right)+\frac{\partial}{\partial y}\left(k(x, y) \frac{\partial T}{\partial y}\right) \\
& +P(\mathbf{x}, t, T)
\end{aligned}
$$

with

$P(\mathbf{x}, t, T)=-u(x, y) w(x, y) \frac{\partial T}{\partial z}+k(x, y) \frac{\partial^{2} T}{\partial z^{2}}$

with boundary conditions, already made homogeneous in the $x$ and $y$ directions, given by:

$$
\begin{aligned}
& {\left[\alpha+\beta k(x, y) \frac{\partial}{\partial \mathbf{n}}\right] T(x, y, z, t)=0, \quad x, y \in S_{x y},} \\
& 0<z<L_{z}, \quad t>0
\end{aligned}
$$

where $S_{x y}$ refers to the outside wall surface, where the boundary conditions can be of first, second or third kind, depending on the chosen values of the coefficients $\alpha$ and $\beta$. The initial condition is written in general form as:

$T(x, y, z, t=0)=f(x, y, z), \quad 0<x<L_{x}, \quad 0<y<L_{y}, \quad 0<z<L_{z}$

while the boundary conditions in the longitudinal direction are taken in the present case as:

$T(x, y, z=0, t)=T_{\text {in }}(x, y),\left.\frac{\partial T}{\partial z}\right|_{z=L_{z}}=0, \quad 0<x<L_{x}, \quad 0<y<L_{y}, \quad t>0$

The space variable coefficients are given by:

$u(x, y)= \begin{cases}u_{f}(x, y), & \text { within the channel (fluid) } \\ u_{s} \rightarrow 0, & \text { within the wall (solid) }\end{cases}$

$w(x, y)= \begin{cases}w_{f}, & \text { within the channel (fluid) } \\ w_{s}, & \text { within the wall (solid) }\end{cases}$

$k(x, y)= \begin{cases}k_{f}, & \text { within the channel (fluid) } \\ k_{s}, & \text { within the wall (solid) }\end{cases}$

One may observe that the terms with partial derivatives with respect to the coordinate $z$ were represented within the source term $P(\mathbf{x}, t, T)$, already reflecting the choice of adopting the partial transformation scheme in this case, i.e., to eliminate by integral transformation only the coordinates $x$ and $y$, in which the diffusive effects are predominant, and not applying the integral transformation over the coordinate $z$.

Hence, In solving problem (11) via the GITT, the following integral transform pair is adopted:

$\bar{T}_{i}(z, t)=\int_{0}^{L_{y}} \int_{0}^{L_{x}} w(x, y) \tilde{\psi}_{i}(x, y) T(x, y, z, t) d x d y$ transform

$T(x, y, z, t)=\sum_{i=1}^{\infty} \tilde{\psi}_{i}(x, y) \bar{T}_{i}(z, t) \quad$ inverse 
where the eigenfunctions $\tilde{\psi}_{i}(x, y)$ and corresponding eigenvalues $\mu_{i}$ are obtained from the solution of the following eigenvalue problem:

$\frac{\partial}{\partial x}\left(k(x, y) \frac{\partial \psi_{i}(x, y)}{\partial x}\right)+\frac{\partial}{\partial y}\left(k(x, y) \frac{\partial \psi_{i}(x, y)}{\partial y}\right)+\mu_{i}^{2} w(x, y) \psi_{i}=0$

$\left[\alpha+\beta k(x, y) \frac{\partial}{\partial \mathbf{n}}\right] \psi_{i}(x, y)=0, \quad x, y \in S_{x y}$

with normalized eigenfunctions and norms written as:

$\tilde{\psi}_{i}(x, y)=\frac{\psi_{i}(x, y)}{\sqrt{N_{i}}}, \quad N_{i}=\int_{0}^{L_{y}} \int_{0}^{L_{x}} w(x, y) \psi_{i}^{2}(x, y) d x d y$

Again, the GITT itself is employed in the solution of problem (13), yielding analytical representations for the eigenfunctions, after the integral transformation process reduces it to an algebraic eigenvalue problem (see Appendix A). Problem (11) is then integral transformed through the operator $\int_{0}^{L_{y}} \int_{0}^{L_{x}} \tilde{\psi}_{i}(x, y)(\cdot) d x d y$, yielding the one-dimensional partial differential transformed system:

$\frac{\partial \bar{T}_{i}(z, t)}{\partial t}+\mu_{i}^{2} \bar{T}_{i}=\bar{g}_{i}(z, t, \overline{\mathbf{T}}), \quad i=1,2, \ldots$

$$
\begin{aligned}
\bar{g}_{i}(z, t, \overline{\mathbf{T}})= & -\sum_{j=1}^{\infty} \frac{\partial \bar{T}_{j}}{\partial z} \int_{0}^{L_{y}} \int_{0}^{L_{x}} w(x, y) u(x, y) \tilde{\psi}_{i}(x, y) \tilde{\psi}_{j}(x, y) d x d y \\
& -\sum_{j=1}^{\infty} \frac{\partial^{2} \bar{T}_{j}}{\partial z^{2}} \int_{0}^{L_{y}} \int_{0}^{L_{x}} k(x, y) \tilde{\psi}_{i}(x, y) \tilde{\psi}_{j}(x, y) d x d y
\end{aligned}
$$

where

$\overline{\mathbf{T}}=\left\{\bar{T}_{1}, \bar{T}_{2}, \ldots\right\}$

with the transformed boundary and initial conditions:

$\bar{T}_{i}(z=0, t)=\bar{T}_{i n, i} \equiv \int_{0}^{L_{y}} \int_{0}^{L_{x}} w(x, y) \tilde{\psi}_{i}(x, y) T_{i n}(x, y) d x d y,\left.\quad \frac{\partial \bar{T}_{i}}{\partial z}\right|_{z=L_{z}}=0$

$\bar{T}_{i}(z, t=0)=\bar{f}_{i}(z) \equiv \int_{0}^{L_{y}} \int_{0}^{L_{x}} w(x, y) \tilde{\psi}_{i}(x, y) f(x, y, z) d x d y$

The partially transformed system given by Eq. (15), upon truncation to a user prescribed finite order $N$, is numerically solved for the transformed potentials $\bar{T}_{i}(z, t)$, with $i=1,2, \ldots, N$. Then, the inversion formula given by Eq. (12b), can be directly employed to recover the original temperature field $T(x, y, z, t)$.

\section{Results and discussion}

The first step in the present analysis is the comparison of the flow problem integral transformation in a single domain, against an exact analytical solution. A quite simple case is then first investigated, consisting of the flow inside a circular channel (HagenPoiseuille flow). For the definition of a single region model defined in a rectangular domain, consider Fig. 3, where it is depicted the circular channel with radius $R=1$ inside a square with side $2 R$. In Fig. 3, the gray filled region is considered to be the solid channel walls, whereas the blank region is considered to be the region of the fluid stream. This cross section is supposed to remain constant along the flow direction. In the same figure, it can also be observed the lines $r_{1}$ and $r_{2}$, which are going to be used later for plotting the velocity profiles.

As an illustration, consider $\Delta p / L_{z}=-4, v_{f}=1$ and $\rho_{f}=1$, then the exact analytical solution for the fully developed velocity field inside the circular duct is given by:

$u(r)=1-r^{2}$

which is going to be used as a benchmark for verification purposes.

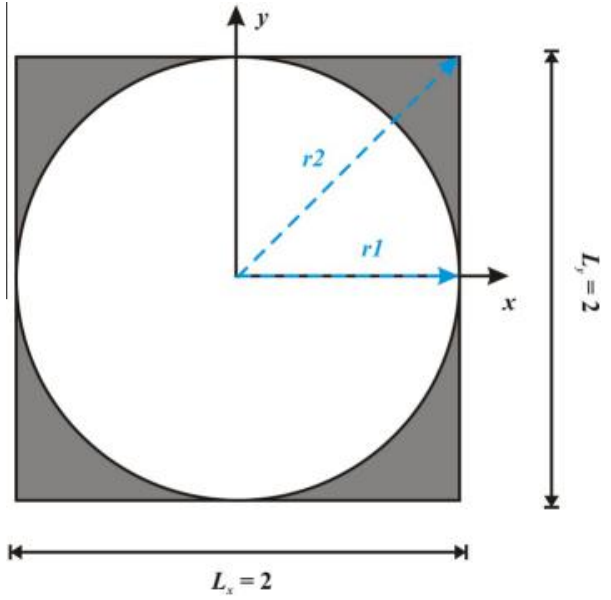

Fig. 3. Schematic representation of the domain cross section (flow in circular tube) employed in the single domain formulation - the grey filled regions correspond to solid regions and the blank region corresponds to the fluid flow region.

For the solution through the single domain methodology described in Section 2, besides the pressure difference and the fluid properties, already defined above, the following values have been assigned to the parameters related to the solid region: $v_{s}=10^{2}$ and $\rho_{s}=\rho_{f}$. Tables $1 \mathrm{a}$ and $1 \mathrm{~b}$ illustrate the convergence behavior of the velocity field, presenting the calculated velocity $u$ at some different positions along directions $r_{1}$ and $r_{2}$ (defined in Fig. 3), respectively, by varying the truncation order in the velocity eigenfunction expansion, $N=50,65,80,95,110$, with a fixed number of terms in the solution of the eigenvalue problem $(M=120)$. It is observed a convergence of at least three significant digits with the increase in the truncation order, and an agreement of at least two significant digits with the exact velocities at the selected positions, calculated from Eq. (16). It is inspected that in the worst case the relative error remains below $3 \%$, which occurs near the fluidsolid interface.

So as to have an overall picture of the approximate solution for the flow problem obtained through the single domain formulation, in Figs. 4a and 4b this approximate solution is critically compared to the exact analytical velocity profiles along the two directions $r_{1}$ and $r_{2}$ (defined in Fig. 3), respectively, where an excellent agreement between the approximate and exact results is observed throughout, even for the region near the interface between the fluid and solid regions. The figures also clearly show that the solid region presents zero velocity, as it should be, and the no-slip condition at the circular contour corresponding to the channel walls is satisfied. Agreement may also be improved by employing an integral balance approach to rewrite the eigenfunction expansions with improved convergence, as described in more details in [8].

Table 1a

Convergence behavior of the velocity field (along $r_{1}$ ) with respect to the truncation order of velocity field expansion $(N)$, with fixed $M=120$ terms in the eigenvalue problem solution (flow in circular tube).

\begin{tabular}{lllll}
\hline$N$ & $u$ & & & \\
\cline { 2 - 5 } & $r_{1}=0.2$ & $r_{1}=0.4$ & $r_{1}=0.6$ & $r_{1}=0.8$ \\
\hline$N=50$ & 0.9600 & 0.8365 & 0.6326 & 0.3497 \\
$N=65$ & 0.9594 & 0.8366 & 0.6321 & 0.3493 \\
$N=80$ & 0.9599 & 0.8363 & 0.6322 & 0.3492 \\
$N=95$ & 0.9599 & 0.8368 & 0.6326 & 0.3498 \\
$N=110$ & 0.9596 & 0.8367 & 0.6326 & 0.3498 \\
Exact & 0.96 & 0.84 & 0.64 & 0.36 \\
\hline
\end{tabular}


Table 1b

Convergence behavior of the velocity field (along $r_{2}$ ) with respect to the truncation order of velocity field expansion $(N)$, with fixed $M=120$ terms in the eigenvalue problem solution (flow in circular tube).

\begin{tabular}{lllll}
\hline$N$ & $u$ & & & \\
\cline { 2 - 5 } & $r_{2}=0.2$ & $r_{2}=0.4$ & $r_{2}=0.6$ & $r_{2}=0.8$ \\
\hline$N=50$ & 0.9591 & 0.8362 & 0.6393 & 0.3669 \\
$N=65$ & 0.9588 & 0.8357 & 0.6392 & 0.3665 \\
$N=80$ & 0.9592 & 0.8361 & 0.6389 & 0.3663 \\
$N=95$ & 0.9590 & 0.8359 & 0.6390 & 0.3663 \\
$N=110$ & 0.9589 & 0.8361 & 0.6392 & 0.3664 \\
Exact & 0.96 & 0.84 & 0.64 & 0.36 \\
\hline
\end{tabular}

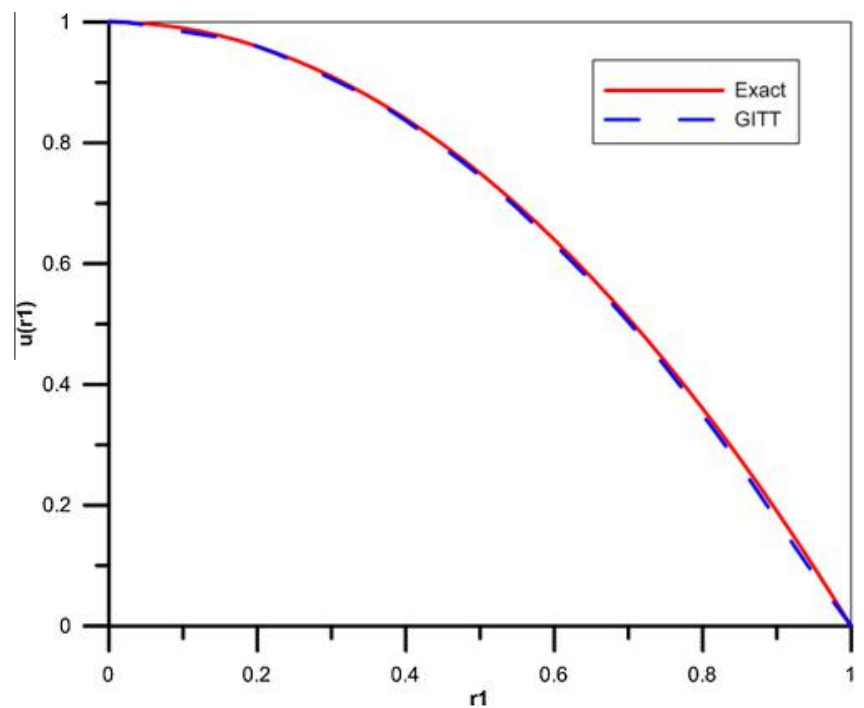

Fig. 4a. Comparison between exact and single domain formulation solution (flow in circular tube) - velocity profile along $r_{1}$.

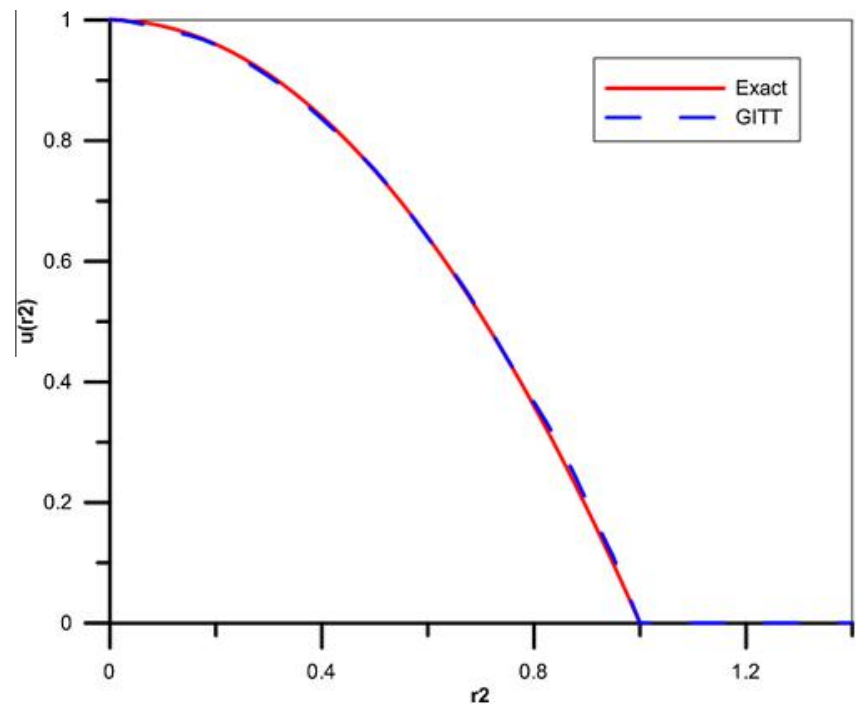

Fig. 4b. Comparison between exact and single domain formulation solution (flow in circular tube) - velocity profile along $r_{2}$.

In order to further illustrate the solution methodology herein presented, it is proposed the investigation of the flow in a channel with the cross-section shape presented in Fig. 5, which also defines the single region model in a rectangular domain, where the grey filled region is considered to be the region of solid walls, whereas the blank region is considered to be the region of the fluid stream, where the fully developed velocity field is first to be determined. For this case, since there is no exact solution available, the problem was also modeled on the commercial CFD solver COMSOL Multiphysics [4], in order to provide a numerical solution through the finite element method for comparison purposes.

In Figs. $6 a$ and $6 b$, the GITT solution of the flow problem is critically compared to the velocity profiles obtained through the COMSOL Multiphysics [4] software along $y$ (for $x=0,0.3,0.6$ ) and along $x$ (for $y=0.3,0.5,0.8$ ), respectively, and an excellent agreement between the hybrid and numerical solutions is observed throughout. Again, it becomes evident that the single domain formulation solution recovers the expected null velocity fields at the solid region, also respecting the no-slip boundary condition, while allowing for a more straightforward integral transformation of the complex configuration, by enveloping the irregular channel within a simpler regular domain with the help of the space variable coefficients.

The analysis of the conjugated heat transfer problem is now considered, for the proposed geometry of a laser ablated microchannel on a polymeric substrate, as presented in Fig. 7. A particular numerical case with $T_{i n}=0$ and external walls boundary

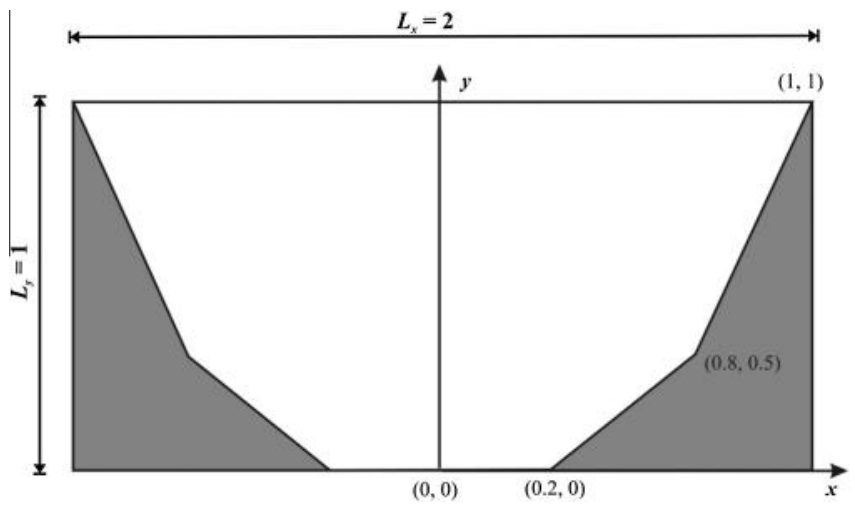

Fig. 5. Schematic representation of the domain cross section employed in the single domain formulation - the grey filled regions correspond to solid regions and the blank region corresponds to the fluid flow region.

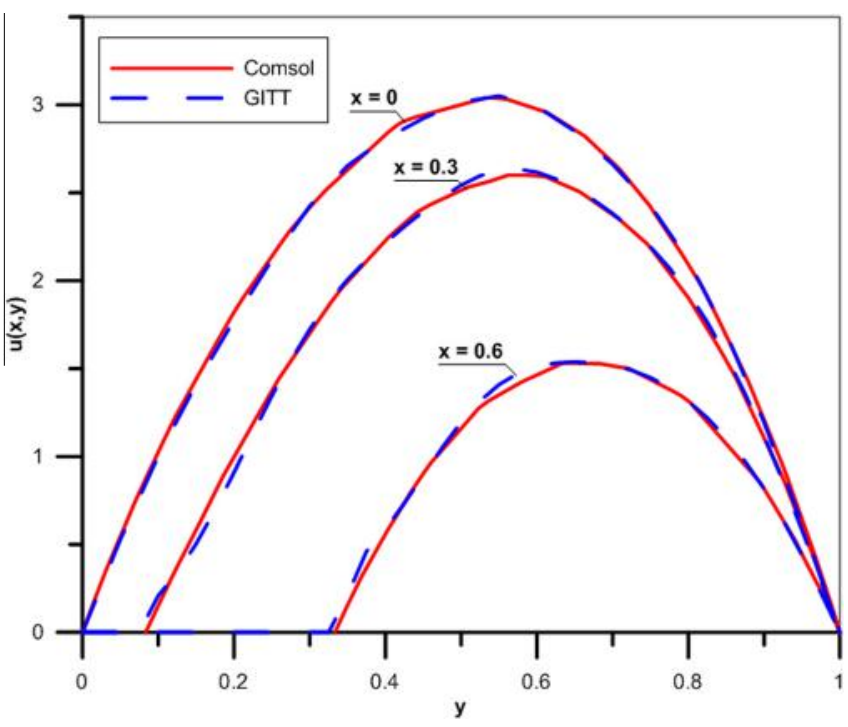

Fig. 6a. Comparison between finite elements solution (COMSOL [4]) and GITT with single domain formulation solution for flow in irregular channel - velocity profiles along $y$. 


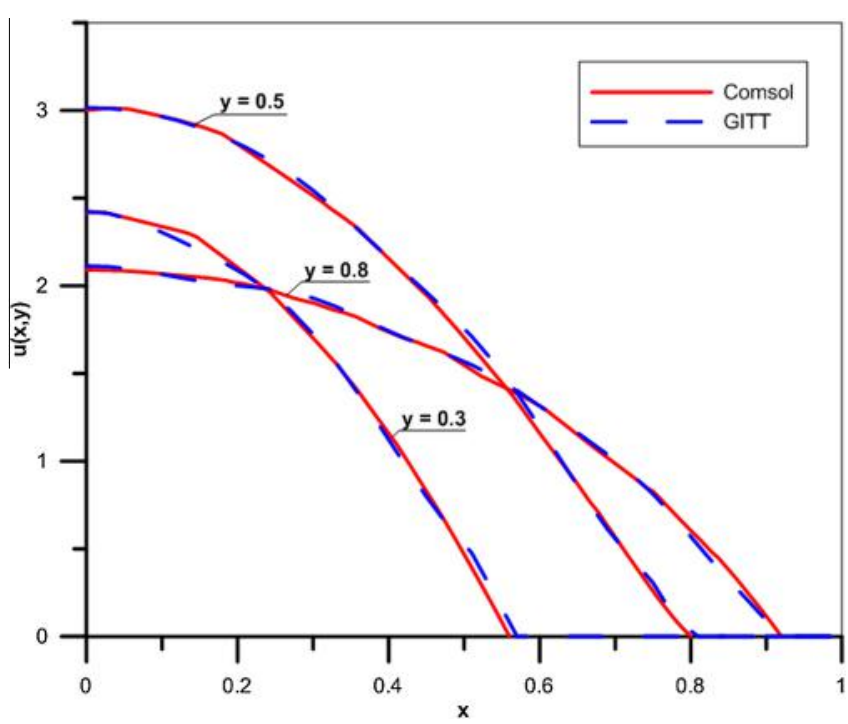

Fig. 6b. Comparison between finite elements solution (COMSOL [4]) and GITT with single domain formulation solution for flow in irregular channel - velocity profiles along $x$.

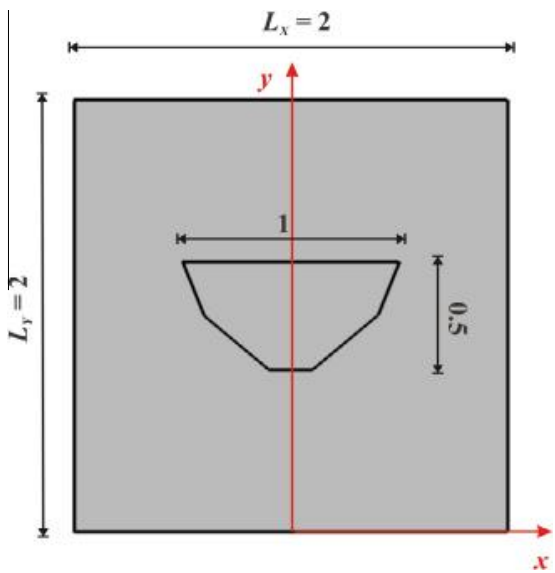

Fig. 7. Schematic representation of micro-channel on polymeric substrate for analysis of conjugated heat transfer in irregular channel and surrounding substrate.

condition $T=1$ ( $\alpha=1$ and $\beta=0$ ), dimensionless solid thermal conductivity, $k_{s}=0.25$, and fluid thermal conductivity, $k_{f}=1$, is more closely analyzed to report a few representative results.

Fig. 8(a) provides the temperature distributions along the $x$ coordinate at $y=1$, from the centerline of the channel up to the external wall surface, while Fig. 8(b) shows the temperature distributions along the vertical $y$ coordinate at the centerline $x=0$, all the way from the bottom to the top base of the polymeric structure. Again, the agreement of the hybrid GITT solution with the purely numerical solution of the COMSOL system [4] is remarkable, all along the thermally developing region, as can be seen from the various curves for different values of the longitudinal coordinate, $z$. A tabular inspection for selected positions along the $x$ axis $(x=0$, $0.3,0.6$, and 0.9 ) for $y=1$; and for selected positions along the $y$ axis ( $y=1,1.3,1.6$, and 1.9 ), for $x=0$, indicated that the maximum deviation between the two solutions was below $1.5 \%$ in the worst case.

A comparison of the GITT and the COMSOL [4] solutions is also provided along the actual transient behavior of the conjugated problem, by showing in Fig. 9 the time evolution of the channel centerline temperature $(x=0, y=1)$, for different values of the longitudinal coordinate, $z$. Again, the curves from the hybrid and

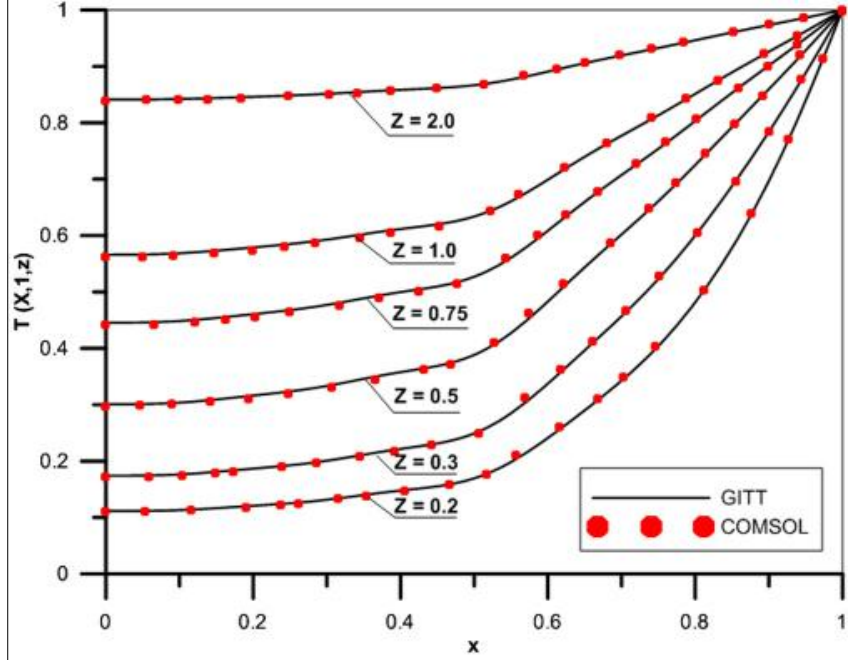

(a)

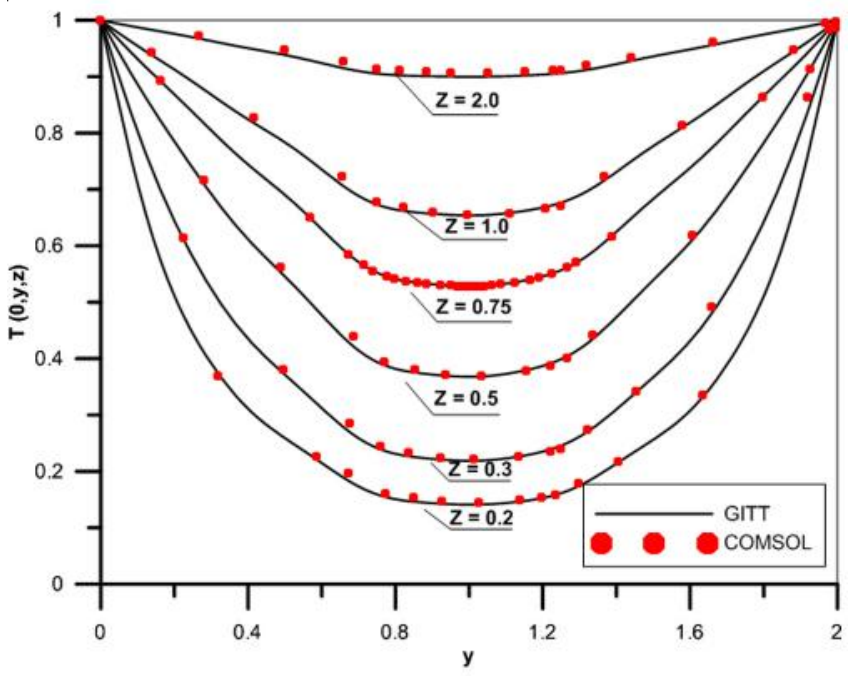

(b)

Fig. 8. Comparison of GITT and numerical (COMSOL Multiphysics [4]) solutions of conjugated heat transfer in complex configuration. (a) temperature distributions along $x$; (b) temperature distributions along $y$.

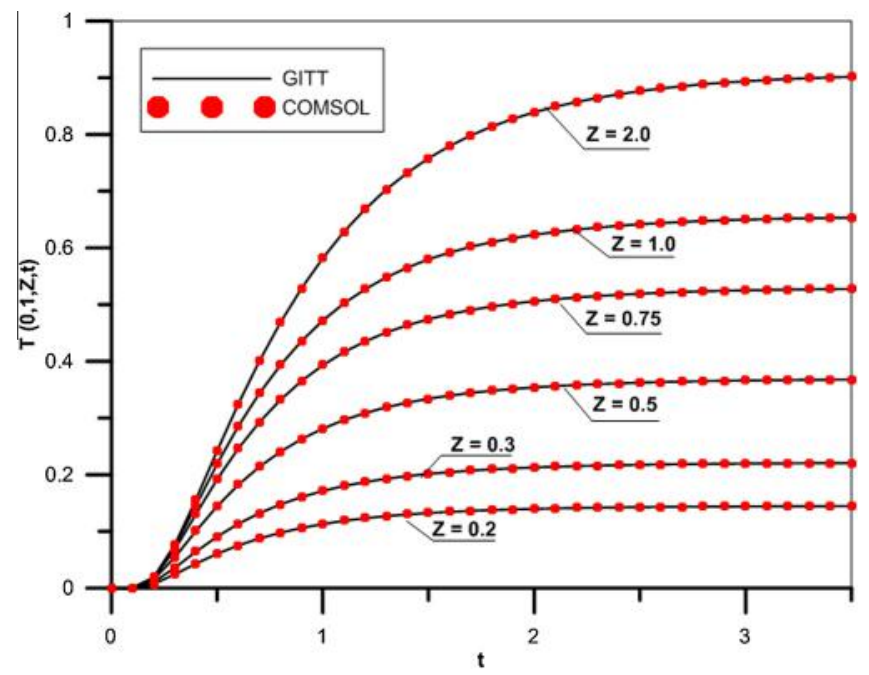

Fig. 9. Comparisons between GITT and COMSOL [4] solutions of the channel centerline temperature $(x=0, y=1)$ as a function of time $(t)$, and for different longitudinal positions $(z)$ 
numerical solutions are practically coincident to the graphical scale, throughout both the time and longitudinal coordinate domains.

Aiming at further illustrating the robustness and flexibility of the approach consolidated in this work, an additional example is provided by considering a multi-stream heat exchanger with five microchannels, as shown in Fig. 10. The wall and fluid temperature profiles are plotted in Fig. 11(a) and (b), comparing the GITT and COMSOL [4] solutions for different longitudinal positions $(z),(a)$

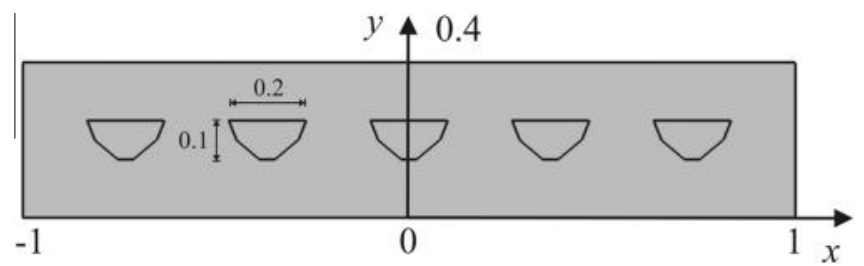

Fig. 10. Schematic representation of the multi-stream heat exchanger cross section.

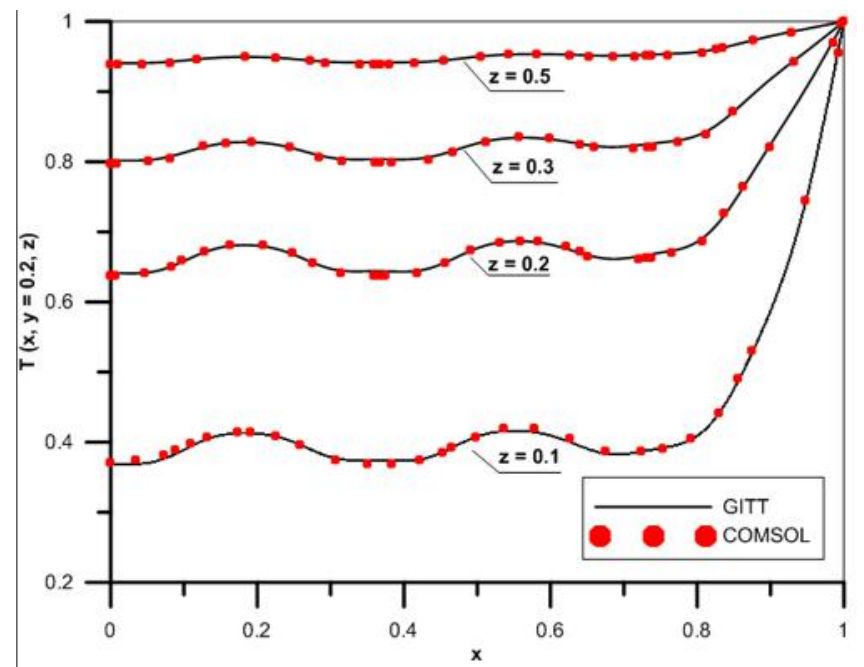

(a)

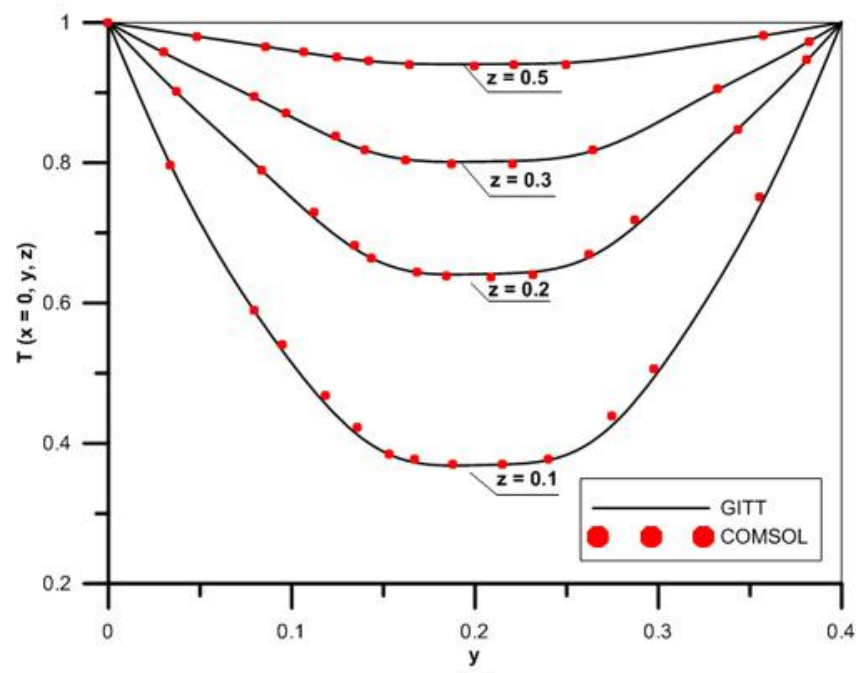

(b)

Fig. 11. Comparisons between GITT and COMSOL [4] solutions for the fluid and wall temperature profiles: (a) along $x$, at $y=0.2$, and (b) along $y$ at $x=0$, for different longitudinal positions $(z)$, for the multi-stream heat exchanger.
Table 2a

Convergence behavior of the steady state temperature profile along $x$ with respect to the truncation order of the expansion $(N)$, with fixed $M=120$ terms in the eigenvalue problem solution (multi-stream heat exchanger), at $z=0.1$.

\begin{tabular}{lllll}
\hline$N$ & \multicolumn{4}{l}{$T(x, y=0.2, z=0.1)$} \\
\cline { 2 - 5 } & $x=0.0$ & $x=0.3$ & $x=0.6$ & $x=0.9$ \\
\hline$N=75$ & 0.3703 & 0.3794 & 0.4076 & 0.5869 \\
$N=85$ & 0.3700 & 0.3792 & 0.4073 & 0.5867 \\
$N=95$ & 0.3698 & 0.3798 & 0.4085 & 0.5867 \\
$N=105$ & 0.3695 & 0.3811 & 0.4084 & 0.5867 \\
$N=115$ & 0.3687 & 0.3786 & 0.4094 & 0.5867 \\
COMSOL [4] & 0.37 & 0.38 & 0.41 & 0.59 \\
\hline
\end{tabular}

Table 2b

Convergence behavior of the steady state temperature profile along $x$ with respect to the truncation order of the expansion $(N)$, with fixed $M=120$ terms in the eigenvalue problem solution (multi-stream heat exchanger), at $z=0.2$.

\begin{tabular}{lllll}
\hline$N$ & \multicolumn{4}{l}{$T(x, y=0.2, z=0.2)$} \\
\cline { 2 - 5 } & $x=0.0$ & $x=0.3$ & $x=0.6$ & $x=0.9$ \\
\hline$N=75$ & 0.6424 & 0.6488 & 0.6808 & 0.8257 \\
$N=85$ & 0.6421 & 0.6486 & 0.6803 & 0.8248 \\
$N=95$ & 0.6421 & 0.6489 & 0.6809 & 0.8248 \\
$N=105$ & 0.6418 & 0.6497 & 0.6808 & 0.8249 \\
$N=115$ & 0.6411 & 0.6474 & 0.6817 & 0.8249 \\
COMSOL [4] & 0.64 & 0.64 & 0.68 & 0.82 \\
\hline
\end{tabular}

Table 3a

Convergence behavior of the steady state temperature profile along $y$ with respect to the truncation order of the expansion $(N)$, with fixed $M=120$ terms in the eigenvalue problem solution (multi-stream heat exchanger), at $z=0.1$.

\begin{tabular}{llll}
\hline$N$ & \multicolumn{2}{l}{$T(x=0, y, z=0.1)$} & $y=0.3$ \\
\cline { 2 - 4 } & $y=0.1$ & $y=0.2$ & 0.5010 \\
\hline$N=75$ & 0.5151 & 0.3703 & 0.5007 \\
$N=85$ & 0.5147 & 0.3700 & 0.5013 \\
$N=95$ & 0.5150 & 0.3699 & 0.5025 \\
$N=105$ & 0.5146 & 0.3695 & 0.5022 \\
$N=115$ & 0.5145 & 0.3688 & 0.51 \\
COMSOL [4] & 0.51 & 0.37 & \\
\hline
\end{tabular}

Table 3b

Convergence behavior of the steady state temperature profile along $y$ with respect to the truncation order of the expansion $(N)$, with fixed $M=120$ terms in the eigenvalue problem solution (multi-stream heat exchanger), at $z=0.2$.

\begin{tabular}{llll}
\hline$N$ & \multicolumn{2}{l}{$T(x=0, y, z=0.2)$} & \\
\cline { 2 - 4 } & $y=0.1$ & $y=0.2$ & $y=0.3$ \\
\hline$N=75$ & 0.7504 & 0.6424 & 0.7382 \\
$N=85$ & 0.7505 & 0.6421 & 0.7384 \\
$N=95$ & 0.7505 & 0.6421 & 0.7386 \\
$N=105$ & 0.7502 & 0.6418 & 0.7393 \\
$N=115$ & 0.7501 & 0.6411 & 0.7391 \\
COMSOL [4] & 0.75 & 0.64 & 0.74 \\
\hline
\end{tabular}

along $x$, at $y=0.2$, and (b) along $y$ at $x=0$. Both across the thickness of the micro-system and along its width, taking just half of the width due to symmetry, one may observe the perfect adherence to the graphical scale between the two sets of temperature results. Clearly, the transitions between the solid and fluid regions are accurately accounted for by the single domain formulation and its corresponding eigenfunctions. It should be highlighted that this problem employs the same entrance and boundary conditions previously used, i.e. $T_{\text {in }}=0$ and $T=1(\alpha=1$ and $\beta=0)$, and dimension- 
less solid thermal conductivity, $k_{\mathrm{s}}=0.25$, and fluid thermal conductivity, $k_{f}=1$. Thus, the multi-stream heat exchanger problem can be modeled as a single domain by properly defining the space variable coefficients so as to capture the five microchannels geometry.

Besides the remarkable adherence between the hybrid and numerical solutions observed in Fig. 11, Tables 2 and 3 illustrate the convergence behavior of the GITT solution with respect to the number of terms employed in the temperature field expansion, where it can be observed a convergence of two significant digits within the truncation orders considered, while the adherence to the COMSOL [4] solution can be confirmed in tabular form.

\section{Conclusions}

The Generalized Integral Transform Technique in conjunction with a single domain formulation strategy has been advanced to propose a procedure for obtaining hybrid numerical-analytical solutions to fluid flow and conjugated heat transfer problems in multiple coupled regions and irregularly shaped domains. The GITT itself is employed in the solution of the auxiliary eigenvalue problem that provides the basis for the eigenfunction expansions of the velocity field and temperature distributions. The methodology is here illustrated in determining the fully developed velocity profile and temperature distribution for a conjugated heat transfer problem in a complex configuration, involving laminar flow in an irregularly shaped channel with the surrounding substrate, followed by the solution of a more complex configuration of a multichannel exchanger. Excellent agreement was achieved between the proposed hybrid solutions and the finite element numerical solutions obtained through the well-known commercial CFD solver COMSOL Multiphysics [4], demonstrating the adequacy of the general approach herein proposed, extending the applicability of the integral transforms method. The analytical nature in the advanced methodology is particularly suitable for very computationally intensive tasks such as in optimization procedures, inverse problem analysis and simulation under uncertainty.

\section{Conflict of interest}

None declared

\section{Acknowledgments}

The authors would like to acknowledge the financial support provided by the Brazilian sponsoring agencies CNPq and FAPERJ.

\section{Appendix A. Solution of eigenvalue problems with the Generalized Integral Transform Technique}

The auxiliary eigenvalue problems that provide the basis for the eigenfunction expansions can be efficiently solved through the Generalized Integral Transform Technique itself, as introduced in [6] and successfully employed in several works, such as $[30,35,40]$, just to cite a few recent contributions. The procedure constitutes a very useful way to handle more general eigenvalue problems, such as in multidimensional problems, complex domains, and non-classical systems, and, therefore, is essential for the solution through integral transforms of the problem proposed in this work.

The basic idea is to employ the Generalized Integral Transform Technique formalism to reduce the eigenvalue problem described by partial differential equations into standard algebraic eigenvalue problems, which can be solved by existing routines for matrix eigensystem analysis. Therefore, the eigenfunctions of the original auxiliary problem can be expressed by eigenfunction expansions based on a simpler auxiliary eigenvalue problem, for which exact analytic solutions exist.

Consider the following problem defined in region $V$ and boundary surface $S$ :

$L \psi(\mathbf{x})=\mu^{2} w(\mathbf{x}) \psi(\mathbf{x}), \quad \mathbf{x} \in V$

$B \psi(\mathbf{x})=0, \quad \mathbf{x} \in S$

where the operators $L$ and $B$ are given by:

$L=-\nabla \cdot(k(\mathbf{x}) \nabla)+d(\mathbf{x})$

$B=\alpha(\mathbf{x})+\beta(\mathbf{x}) k(\mathbf{x}) \frac{\partial}{\partial \mathbf{n}}$

where $w(\mathbf{x}), k(\mathbf{x})$, and $d(\mathbf{x})$ are known functions in region $V$, and $\alpha(\mathbf{x})$, $\beta(\mathbf{x})$ are known functions on the boundary surface $S$.

The problem given by Eqs. (A1a)-(A1d) can be rewritten as:

$\hat{L} \psi(\mathbf{x})=(\hat{L}-L) \psi(\mathbf{x})+\mu^{2} w(\mathbf{x}) \psi(\mathbf{x}), \quad \mathbf{x} \in V$

$\hat{B} \psi(\mathbf{x})=(\hat{B}-B) \psi(\mathbf{x}), \quad \mathbf{x} \in S$

where $\hat{L}$ and $\hat{B}$ are the operators given by:

$\hat{L}=-\nabla \cdot(\hat{k}(\mathbf{x}) \nabla)+\hat{d}(\mathbf{x})$

$\hat{B}=\hat{\alpha}(\mathbf{x})+\hat{\beta}(\mathbf{x}) \hat{k}(\mathbf{x}) \frac{\partial}{\partial \mathbf{n}}$

which are employed to select an auxiliary problem:

$\hat{L} \Omega(\mathbf{x})=\lambda^{2} \hat{w}(\mathbf{x}) \Omega(\mathbf{x}), \quad \mathbf{x} \in V$

$\hat{B} \Omega(\mathbf{x})=0, \quad \mathbf{x} \in S$

where $\hat{w}(\mathbf{x}), \hat{k}(\mathbf{x}), \hat{d}(\mathbf{x}), \hat{\alpha}(\mathbf{x})$, and $\hat{\beta}(\mathbf{x})$ are known coefficients in $V$ and $S$, properly chosen so that the eigenvalue problem given by Eqs. (A3a) and (A3b) allows for exact analytic solution for the eigenvalues $\lambda$ and corresponding eigenfunctions $\Omega(\mathbf{x})$.

Therefore, making use of eigenfunctions orthogonality property, problem (A3) allows the definition of the following integral transform pair:

transform : $\quad \bar{\psi}_{i}=\int_{V} \hat{w}(\mathbf{x}) \tilde{\Omega}_{i}(\mathbf{x}) \psi(\mathbf{x}) d V$

inverse : $\quad \psi(\mathbf{x})=\sum_{i=1}^{\infty} \tilde{\Omega}_{i}(\mathbf{x}) \bar{\psi}_{i}$

where

$\tilde{\Omega}_{i}(\mathbf{x})=\frac{\Omega_{i}(\mathbf{x})}{\sqrt{N_{\Omega_{i}}}}, \quad$ with $\quad N_{\Omega_{i}}=\int_{V} \hat{w}(\mathbf{x}) \Omega_{i}^{2}(\mathbf{x}) d V$

Eq. (A2a) is now operated with $\int_{V} \tilde{\Omega}_{i}(\mathbf{x})(\cdot) d V$, to yield the transformed algebraic system:

$$
\begin{aligned}
\lambda_{i}^{2} \bar{\psi}_{i}= & \int_{S} \gamma_{i}(\hat{B}-B) \psi(\mathbf{x}) d S+\int_{V} \tilde{\Omega}_{i}(\mathbf{x})(\hat{L}-L) \psi(\mathbf{x}) d V \\
& +\mu^{2} \int_{V} \tilde{\Omega}_{i}(\mathbf{x}) w(\mathbf{x}) \psi(\mathbf{x}) d V, \quad i=1,2, \ldots \\
\gamma_{i}= & \frac{\tilde{\Omega}_{i}(\mathbf{x})-\hat{k}(\mathbf{x}) \frac{\partial \tilde{\Omega}_{i}(\mathbf{x})}{\partial \mathbf{n}}}{\hat{\alpha}(\mathbf{x})+\hat{\beta}(\mathbf{x})}
\end{aligned}
$$

After introducing the truncated to the Mth term inversion formula, Eq. (A4b), into Eq. (A5a), the resulting system is written in matrix form as:

$$
(\mathbf{A}+\mathbf{C})\{\overline{\boldsymbol{\psi}}\}=\boldsymbol{\mu}^{\mathbf{2}} \mathbf{B}\{\overline{\boldsymbol{\psi}}\}
$$


with the elements of the $\mathrm{M} \times \mathrm{M}$ matrices, and vector $\boldsymbol{\mu}^{2}$ given by:

$A_{i j}=-\int_{S} \gamma_{i}(\hat{B}-B) \tilde{\Omega}_{j}(\mathbf{x}) d S-\int_{V} \tilde{\Omega}_{i}(\mathbf{x})(\hat{L}-L) \tilde{\Omega}_{j}(\mathbf{x}) d V$

$C_{i j}=\lambda_{i}^{2} \delta_{i j}$

$B_{i j}=\int_{V} w(\mathbf{x}) \tilde{\Omega}_{i}(\mathbf{x}) \tilde{\Omega}_{j}(\mathbf{x}) d V$

$\boldsymbol{\mu}^{2}=\left\{\mu_{1}^{2}, \mu_{2}^{2}, \ldots, \mu_{M}^{2}\right\}$

where $\delta_{i j}$ is the Kronecker delta.

By choosing to use the relation

$$
\begin{aligned}
\int_{V} \tilde{\Omega}_{i}(\mathbf{x}) \nabla \cdot\left(\hat{k}(\mathbf{x}) \nabla \tilde{\Omega}_{j}(\mathbf{x})\right) d V= & \int_{S} \hat{k}(\mathbf{x}) \tilde{\Omega}_{i}(\mathbf{x}) \frac{\partial \tilde{\Omega}_{j}(\mathbf{x})}{\partial \mathbf{n}} d S \\
& -\int_{V} \hat{k}(\mathbf{x}) \nabla \tilde{\Omega}_{i}(\mathbf{x}) \cdot \nabla \tilde{\Omega}_{j}(\mathbf{x}) d V
\end{aligned}
$$

the elements of $\mathbf{A}$ can be calculated through the following working formula:

$$
\begin{aligned}
& \int_{S} \frac{\tilde{\Omega}_{i}(\mathbf{x})-\hat{k}(\mathbf{x}) \frac{\partial \tilde{\Omega}_{i}(\mathbf{x})}{\partial \mathbf{n}}}{\hat{\alpha}(\mathbf{x})+\hat{\beta}(\mathbf{x})}\left[(\alpha(\mathbf{x})-\hat{\alpha}(\mathbf{x})) \tilde{\Omega}_{j}(\mathbf{x})+(\beta(\mathbf{x}) k(\mathbf{x})\right. \\
& \left.\quad-\hat{\beta}(\mathbf{x}) k(\mathbf{x})) \frac{\partial \tilde{\Omega}_{j}(\mathbf{x})}{\partial \mathbf{n}}\right] d S-\int_{S}(k(\mathbf{x})-\hat{k}(\mathbf{x})) \tilde{\Omega}_{i}(\mathbf{x}) \frac{\partial \tilde{\Omega}_{j}(\mathbf{x})}{\partial \mathbf{n}} d S \\
& \quad+\int_{V}(k(\mathbf{x})-\hat{k}(\mathbf{x})) \nabla \tilde{\Omega}_{i}(\mathbf{x}) \cdot \nabla \tilde{\Omega}_{j}(\mathbf{x}) d V \\
& \quad+\int_{V}(d(\mathbf{x})-\hat{d}(\mathbf{x})) \tilde{\Omega}_{i}(\mathbf{x}) \tilde{\Omega}_{j}(\mathbf{x}) d V
\end{aligned}
$$

Therefore, the eigenvalue problem given by Eqs. (A1a) and (A1b) is reduced to the standard algebraic eigenvalue problem given by Eq. (A6a), which can be solved with existing software for matrix eigensystem analysis, directly yielding the eigenvalues $\mu$, whereas the corresponding calculated eigenvectors from this numerical solution, $\bar{\psi}_{i}$, are to be used in the inversion formula, given by Eq. (A4b), to find the desired eigenfunction. By increasing the number of terms in the truncated expansion, one can obtain the results with prescribed accuracy.

\section{References}

[1] W.J. Minkowycz, E.M. Sparrow, J.Y. Murthy, Handbook of Numerical Heat Transfer, second ed., Wiley, New York, 2006.

[2] R.H. Pletcher, J.C. Tannehill, D.A. Anderson, Computational Fluid Mechanics and Heat Transfer, third ed., CRC Press, 2013.

[3] Y. Jaluria, K.E. Torrance, Computational Heat Transfer, Taylor \& Francis, 2003.

[4] COMSOL Multiphysics, CFD and Heat Transfer Modules, (n.d.), <http:/ www.br.comsol.com/products>.

[5] ANSYS Fluent \& ANSYS CFX, Computational Fluid Dynamics (CFD) Software, (n.d.), <http://www.ansys.com/Products/Simulation+Technology/Fluid+ Dynamics>.

[6] R.M. Cotta, Integral Transforms in Computational Heat and Fluid Flow, CRC Press, Boca Raton, 1993.

[7] R.M. Cotta, Benchmark results in computational heat and fluid flow: the integral transform method, Int. J. Heat Mass Transfer 37 (1994) 381-393.

[8] R.M. Cotta, M.D. Mikhailov, Heat Conduction: Lumped Analysis, Integral Transforms, Symbolic Computation, Wiley, Chichester, 1997.

[9] R.M. Cotta, M.D. Mikhailov, Hybrid methods and symbolic computations, in: W.J. Minkowycz, E.M. Sparrow, J.Y. Murthy (Eds.), Handbook of Numerical Heat Transfer, Wiley, New York, 2006.

[10] R.O.C. Guedes, R.M. Cotta, N.C.L. Brum, Heat transfer in laminar tube flow with wall axial conduction effects, J. Thermophys. Heat Transfer 5 (1991) 508-513.

[11] R.O.C. Guedes, M.N. Ozisik, R.M. Cotta, Conjugated periodic turbulent forced convection in a parallel plate channel, J. Heat Transfer 116 (1994) 40-46.

[12] C.P. Naveira, M. Lachi, R.M. Cotta, J. Padet, Hybrid formulation and solution for transient conjugated conduction-external convection, Int. J. Heat Mass Transfer 52 (2009) 112-123.
[13] J.S. Nunes, R.M. Cotta, M. Avelino, S. Kakaç, Conjugated Heat Transfer in Microchannels, in: Microfluid. Based Microsystems Fundam. Appl. NATO Sci. Peace Secur. Ser. A Chem. Biol., Springer, Netherlands, 2010, pp. 61-82.

[14] E. Figueira da Silva, R.M. Cotta, Benchmark results for internal forced convection through integral transformation, Int. Commun. Heat Mass Transfer 23 (1996) 1019-1029.

[15] E. Figueira da Silva, R.M. Cotta, Mixed convection within vertical parallel plates: hybrid solution by integral transforms, Numer. Heat Transfer Part A Appl. 33 (1998) 85-106.

[16] H.A. Machado, R.M. Cotta, Analysis of internal convection with variable physical properties via integral transformation, Numer. Heat Transfer Part A Appl. 36 (1999) 699-724.

[17] S.P.A. Paz, E.N. Macêdo, J.N.N. Quaresma, R.M. Cotta, Eigenfunction expansion solution for boundary-layer equations in cylindrical coordinates: simultaneously developing flow in circular tubes, Numer. Heat Transfer Part A Appl. 52 (2007) 1123-1149.

[18] J.B. Aparecido, R.M. Cotta, M.N. Ozisik, Analytical solutions to two-dimensional diffusion type problems in irregular geometries, J. Franklin Inst. 326 (1989) 421-434.

[19] J.B. Aparecido, R.M. Cotta, Laminar thermally developing flow inside rightangularly triangular ducts, Appl. Sci. Res. 49 (1992) 355-368, http://dx.doi.org/ 10.1007/BF00419981.

[20] J.S. Pérez Guerrero, J.N.N. Quaresma, R.M. Cotta, Simulation of laminar flow inside ducts of irregular geometry using integral transforms, Comput. Mech. 25 (2000) 413-420.

[21] L.A. Sphaier, R.M. Cotta, Analytical and hybrid solutions of diffusion problems within arbitrarily shaped regions via integral transforms, Comput. Mech. 29 (2002) 265-276.

[22] E.R. Monteiro, E.N. Macêdo, J.N.N. Quaresma, R.M. Cotta, Laminar flow and convective heat transfer of non-Newtonian fluids in doubly connected ducts, Int. J. Heat Mass Transfer 53 (2010) 2434-2448.

[23] F. V Castellões, J.N.N. Quaresma, R.M. Cotta, Convective heat transfer enhancement in low reynolds number flows with wavy walls, Int. J. Heat Mass Transfer 53 (2010) 2022-2034

[24] R.L. Silva, J.N.N. Quaresma, C.A.C. Santos, R.M. Cotta, Integral transforms solution for flow development in wavy wall ducts, Int. J. Numer. Methods Heat Fluid Flow 21 (2011) 219-243.

[25] C.B. Sobhan, G.P. Peterson, Microscale and Nanoscale Heat Transfer: Fundamentals and Engineering Applications, CRC Press, Boca Raton, 2008.

[26] N. Kockmann, Transport Phenomena in Micro Process Engineering, SpringerVerlag, Berlin, 2008.

[27] G.L. Morini, Single-phase convective heat transfer in microchannels: a review of experimental results, Int. J. Therm. Sci. 43 (2004) 631-651.

[28] Y. Yener, S. Kakaç, M. Avelino, Okutucu, Single-phase forced convection in microchannels - a state-of-the-art review, in: S. Kakaç, L.L. Vasiliev, Y. Bayazitoglu, Y. Yener (Eds.), Microscale Heat Transf. - Fundam. Appl., 2005.

[29] G. Maranzana, I. Perry, D. Maillet, Mini and microchannels: influence of axial conduction in the walls, Int. J. Heat Mass Transfer 47 (2004) 3993-4004.

[30] D.C. Knupp, C.P. Naveira-Cotta, R.M. Cotta, Theoretical analysis of conjugated heat transfer with a single domain formulation and integral transforms, Int. Commun. Heat Mass Transfer 39 (2012) 355-362.

[31] D.C. Knupp, C.P. Naveira-Cotta, R.M. Cotta, Conjugated convection-conduction analysis in microchannels with axial diffusion effects and a single domain formulation, J. Heat Transfer 135 (2013) 091008.

[32] D.C. Knupp, R.M. Cotta, C.P. Naveira-Cotta, Heat transfer in microchannels with upstream-downstream regions coupling and wall conjugation effects, Numer. Heat Transfer Part B Fundam. 64 (2013) 365-387.

[33] D.C. Knupp, C.P. Naveira-Cotta, R.M. Cotta, Unified integral transforms in single domain formulation for internal flow three-dimensional conjugated problems, in: 15th Int. Heat Transf. Conf. - IHTC-15, August 10-15, 2014.

[34] D.C. Knupp, C.P. Naveira-Cotta, R.M. Cotta, Theoretical-experimental analysis of conjugated heat transfer in nanocomposite heat spreaders with multiple microchannels, Int. J. Heat Mass Transfer 74 (2014) 306-318.

[35] L.A. Sphaier, R.M. Cotta, Integral transform analysis of multidimensional eigenvalue problems within irregular domains, Numer. Heat Transfer Part BFundam. 38 (2000) 157-175.

[36] C.P. Naveira-Cotta, R.M. Cotta, H.R.B. Orlande, O. Fudym, Eigenfunction expansions for transient diffusion in heterogeneous media, Int. J. Heat Mass Transfer 52 (2009) 5029-5039.

[37] S. Wolfram, The Mathematica Book, Wolfram Media, Cambridge, 2005.

[38] R.M. Cotta, D.C. Knupp, C.P. Naveira-Cotta, L.A. Sphaier, J.N.N. Quaresma, The unified integral transforms (UNIT) algorithm with total and partial transformation, Comput. Therm. Sci., in press, http://dx.doi.org/10.1615/ ComputThermalScien.2014008663.

[39] R.M. Cotta, D.C. Knupp, C.P. Naveira-Cotta, L.A. Sphaier, J.N.N. Quaresma, Unified integral transforms algorithm for solving multidimensional nonlinear convection-diffusion problems, Numer. Heat Transfer Part A Appl. 63 (2013) 840-866.

[40] D.C. Knupp, C.P. Naveira-Cotta, JV.C Ayres, R.M. Cotta, H.R.B. Orlande Theoretical-experimental analysis of heat transfer in nonhomogeneous solids via improved lumped formulation, integral transforms and infrared thermography, Int. J. Therm. Sci. 62 (2012) 71-84. 